\title{
(6) Comparison between PET CT and AGO score for operability evaluation in recurrent ovarian cancer: \\ a retrospective study. \\ institutCurie
}

J DELVALLEE ${ }^{1,2,3}$ C CAMBRA ${ }^{1,3,4}$, C BONNEAU1 1,3, N POUGET $^{1,3}$, R ROUZIER, CA PHILIP1 $^{1,3,5}$

${ }^{1}$ Department of Gynecology, Curie Institute, Saint Cloud, France; ${ }^{2}$ Faculty of Medicine, University François Rabelais and CHRU Tours, France; ${ }^{3}$ University Versailles Saint Quentin en Yvelines, Versailles, France; ${ }^{4}$ Faculty of Medicine, University of Occidental Bretagne and CHRU Morvan, Brest, France; ${ }^{5} \mathrm{CHU}$

Croix Rousse, University Lyon 1, Lyon, France

\section{Objectives:}

- to compare positron emission computarized tomography (PET CT) in epithelial recurrent ovarian cancer $(R O C)$ with the validated $A G O$ score to predict the feasibility of secondary cytoreductive surgery (SCS)

- to determine predictive factors of complete cytoreductive surgery and survival.

\section{Material and methods}

We conducted a retrospective multi centric study in Curie Institute (2 sites)

All the patients treated for an epithelial ROC were screened and included if they underwent a PET CT before treatment.

We created a PET-CT assesment that rejected surgery if one of the following items was reported: diffuse carcinomatosis, extra-abdominal lesions, and supradiaphragmatic lymph nodes

An AGO score equal to 3 was considered to support the feasibility of surgery.

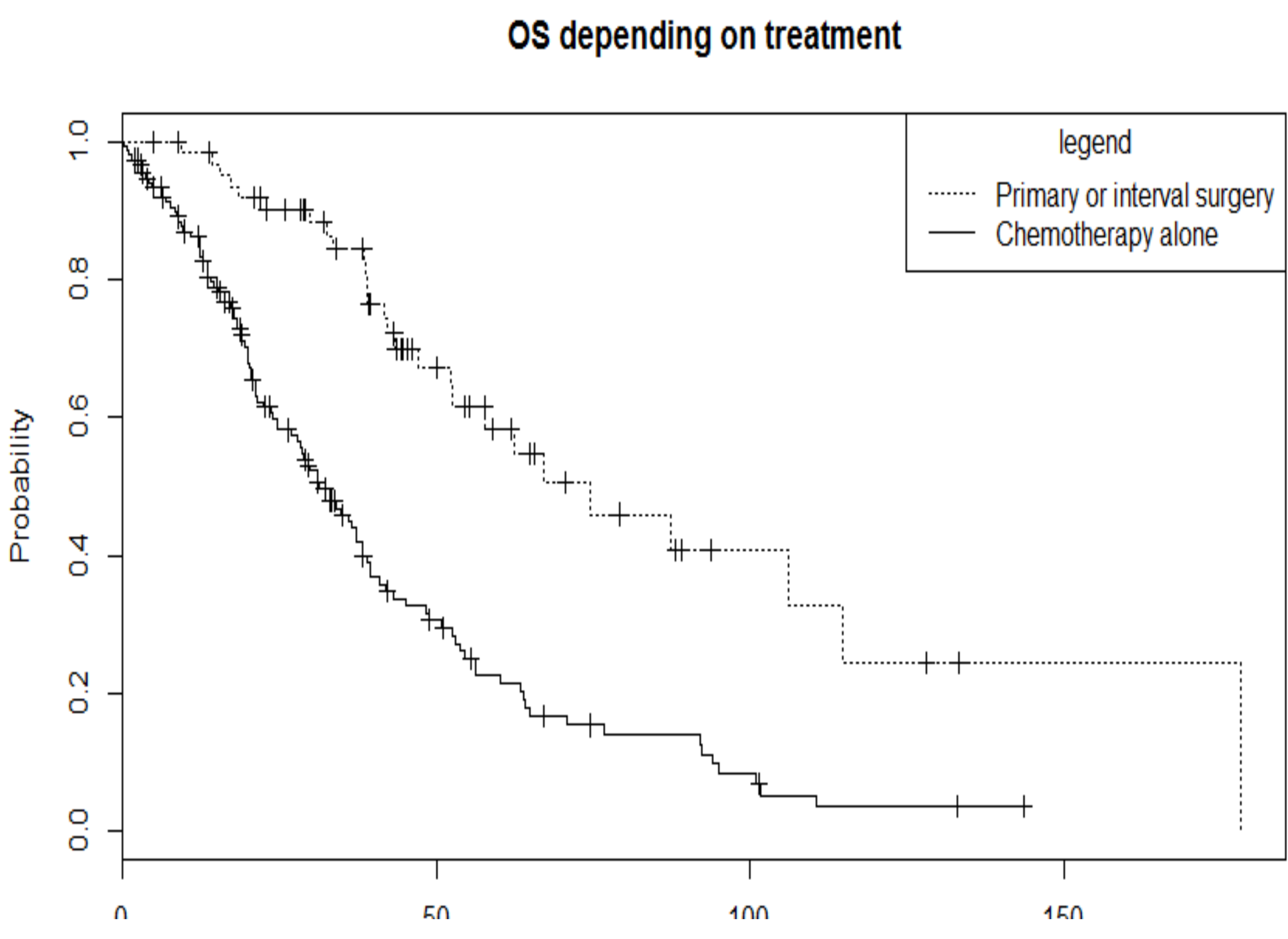

OS based on TEP and AGO scores combination

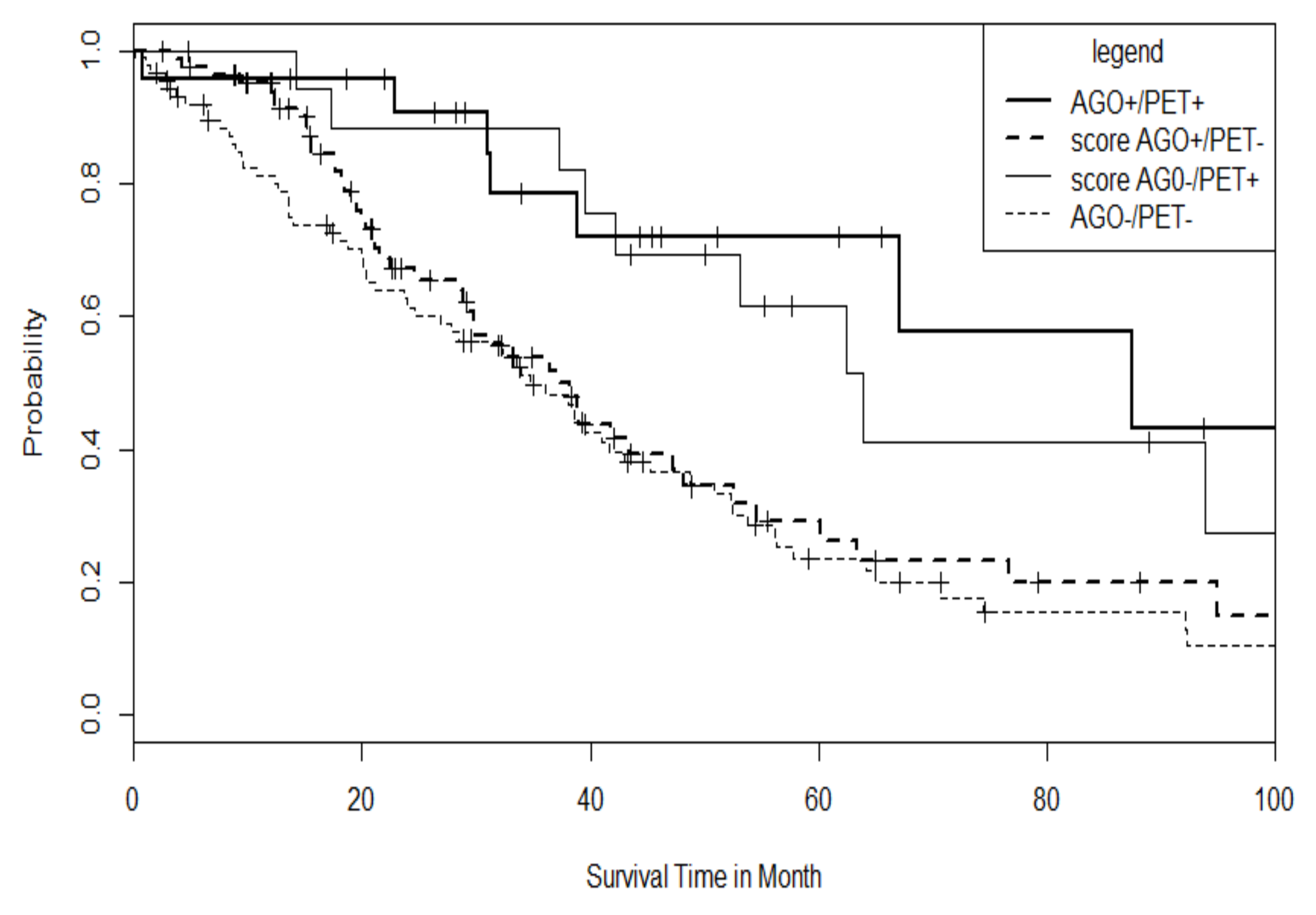

\section{Patients characteristics}

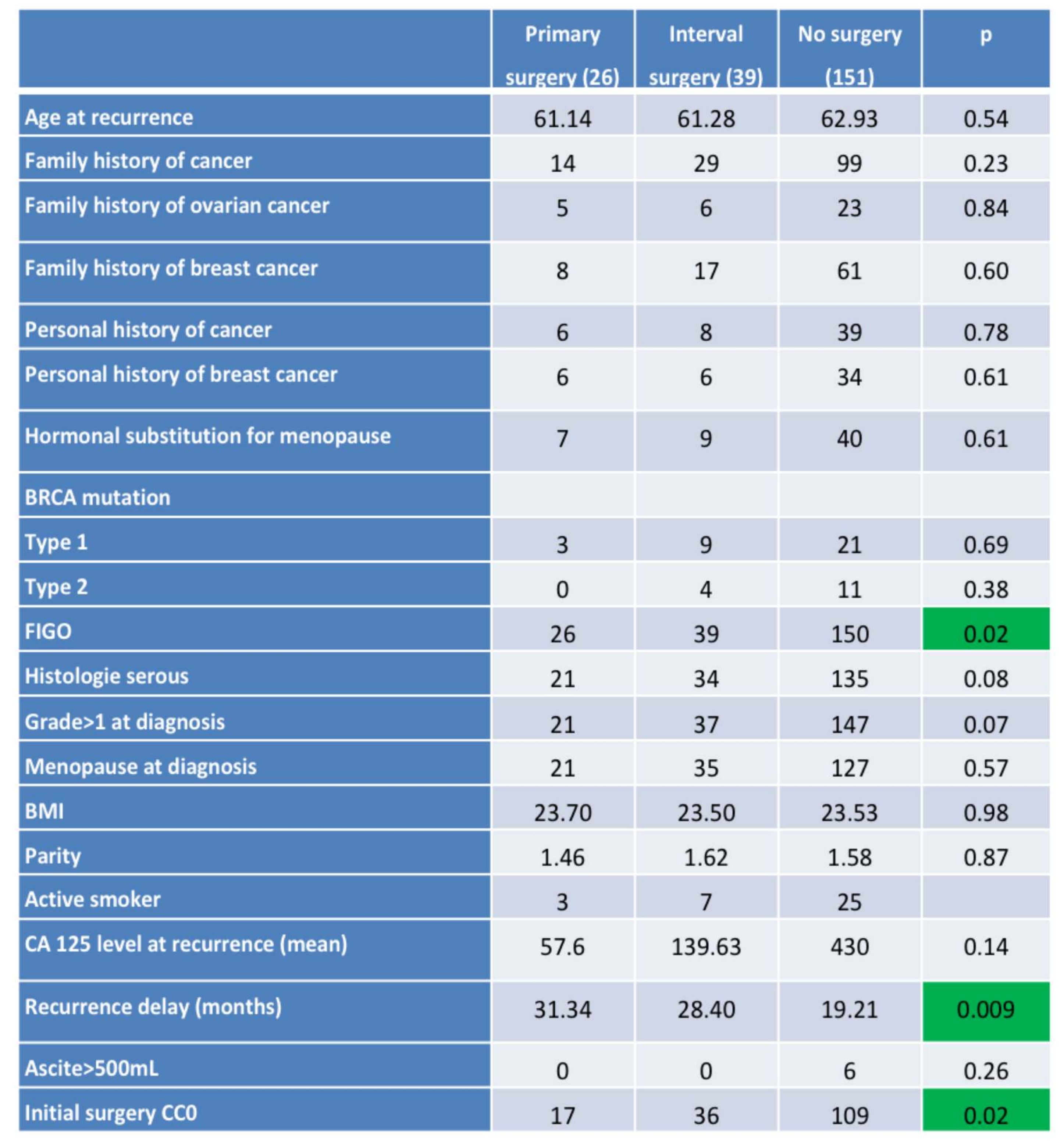

\section{OS on months depending on treatment:}

Median survival is 107 months with SCS vs 27 without $(p=1.18 \mathrm{e}-07$ ).

No follow up after 140 months for the only chemotherapy group.

\section{Results}

546 patients were treated for an epithelial ROC during the study period, among them 216 underwent a PET CT before any treatment.

66patients underwent SCS: complete cytoreduction was achieved in 58 patients $(89.2 \%)$ and no case of mortality was reported within 60 days post-surgery.

In univariate analysis, the following TEP items were statistically different between the 3 groups: diffuse carcinomatosis $(8 / 19 / 102 p=0,0006)$, extra abdominal lesion $(7 / 8 / 82 \mathrm{p}=\mathbf{0 , 0 0 0 1})$ and sus-diaphragmatic nodes $(4 / 4 / 69$ $\mathrm{p}<0,0001)$.

Overall survival was higher in second cytoreduction surgery group than the chemotherapy group.

\section{OS depending on PET and AGO scores combination}

OS is 86 months for AGO+/PET+ group, 62 for AGO-/PET+, 39 for AGO+/PETand 38 for AGO-/PET-.In univariate analysis, AGO score of 3 and PET score of 0 associated were statically associated in OS $(p<a ̀, 01)$

\section{Conclusion}

In our series, the positive AGO score remains a predictor of operability in recurrent epithelial ovarian cancer.

However, the PET TDM and its different composite score criteria are more strongly associated with the feasibility of SCC and can be used in combination with the AGO score because the 2 tests remain significant in multivariate. They are complementary. 